\title{
Class C1 decoy oligodeoxynucleotide inhibits profibrotic genes expression in rat hepatic stellate cells
}

\author{
CHUN RAO ${ }^{1-4 *}$, YI-RAN NI ${ }^{2,3^{*}}$, YAN-MIN ZHAO ${ }^{2,5}$, YAN-QIONG ZHANG ${ }^{2,3,6}$, RUI-TING ZHOU $^{2,3}$, \\ CHANG-BAI LIU ${ }^{2,6}$, LIN HAN ${ }^{1}$ and JIANG-FENG WU ${ }^{1-3,6}$
}

${ }^{1}$ Department of Pathology, The People's Hospital of China Three Gorges University and the First People's Hospital of Yichang, Yichang, Hubei 443000; ${ }^{2}$ Department of Anatomy and Histology, Medical College, China Three Gorges University, Yichang,

Hubei 443002; ${ }^{3}$ Institute of Organ Fibrosis and Targeted Drug Delivery, China Three Gorges University, Yichang,

Hubei 443002; ${ }^{4}$ Department of Internal Medicine, Hospital of Hubei University, Wuhan, Hubei 430062;

${ }^{5}$ Pharmacy Department, Yichang Fifth People's Hospital, Yichang, Hubei 443007; ${ }^{6}$ Hubei Key Laboratory of Tumor

Microenvironment and Immunotherapy, China Three Gorges University, Yichang, Hubei 443002, P.R. China

Received October 5, 2018; Accepted November 7, 2019

DOI: $10.3892 / \mathrm{mmr} .2019 .10881$

\begin{abstract}
The aim of the present study was to investigate whether class $\mathrm{C} 1$ decoy oligodeoxynucleotides (ODNs) can inhibit the expression of pro-fibrotic genes associated with rat hepatic stellate cell (HSC) activation and hepatic fibrosis. Luciferase reporter assays were performed to test the promoter activities of transforming growth factor (TGF)- $\beta$ and its downstream target genes following transfection of decoy ODNs and plasmids into HSC-T6 cells, and western blot assays were performed to measure the protein expression of those genes following decoy ODN transfection. Class C1 decoy ODNs were confirmed to inhibit the promoter activity of TGF- $\beta$ and its downstream target genes, such as type 1 collagen (COLI) 1 , tissue inhibitor of metalloproteinases (TIMP)1 and $\alpha$-smooth muscle actin by Gaussia luciferase reporter assay, and to further downregulate the expression of TGF- $\beta$, SMAD3, COLI 1 and TIMP1 by western blotting in activated HSC-T6 cells. In conclusion, class C1 decoy ODNs inhibited pro-fibrotic gene expression in rat HSCS by downregulating TGF- $\beta$ signaling.
\end{abstract}

Correspondence to: Dr Lin Han, Department of Pathology, The People's Hospital of China Three Gorges University and the First People's Hospital of Yichang, 4 Hudi Road, Yichang, Hubei 443000, P.R. China

E-mail: hanlinfofo2001@sina.com

Professor Jiang-Feng Wu, Department of Anatomy and Histology, Medical College, China Three Gorges University, 8 Daxue Road, Yichang, Hubei 443002, P.R. China

E-mail: jiangfengwu2011@163.com

*Contributed equally

Key words: class $\mathrm{C} 1$ decoy oligodeoxynucleotides, transforming growth factor- $\beta$ signaling, hepatic stellate cells

\section{Introduction}

Hepatic fibrosis is a reversible wound healing process elicited by various damaging factors, such as viruses, parasites and alcohol, all of which lead to liver cell injury accompanied by inflammatory responses. Upon stimulation by a multitude of signals, hepatic stellate cells (HSCs) transform into myofibroblast-like cells, with ensuing excessive production of extracellular matrix (ECM), including type I and type III collagen. Eventually, the disease may progress to liver cirrhosis and even liver cancer $(1,2)$. Therefore, it is crucial to arrest the progression of hepatic fibrosis.

During the process of hepatic fibrosis, the activation of HSCs is fundamental. Once activated, the HSCs transform into myofibroblasts, which characteristically express $\alpha$-smooth muscle actin (SMA). Furthermore, pro-fibrotic factors are generated, such as transforming growth factor (TGF)- $\beta 1$ and tissue inhibitors of metalloproteinases (TIMPs). It was previously demonstrated that TGF- $\beta$ signaling is key to the development of hepatic fibrosis (3) and it enhances the synthesis of hepatic fibrosis-related proteins, such as type I collagen (COLI) $\alpha 1$, COLI 2 and TIMP1 (4-6). Hence, molecular therapy targeting HSCs and inhibiting the TGF- $\beta$ signaling pathway may inhibit HSC activation and may block or even reverse the pathological process of liver fibrosis. It is widely accepted that TGF- $\beta$ canonical signaling is essential for the activation of HSCs; non-canonical signaling, which is associated with multiple different pathways, such as mitogen-activated protein kinase (MAPK), phosphoinositide 3 kinase (PI3K)/protein kinase B (AKT) and Wnt pathways, may also contribute to the activation of HSCs and liver fibrosis $(7,8)$. Recent studies have reported the role of Notch signaling in hepatic fibrosis and the crosstalk between Notch and TGF- $\beta$ signaling. There is evidence that the expression of major components of the Notch signaling pathway, including Notch3, Jagged1 and the downstream transcription factor (TF) Hairy and Enhancer of Split 1 (HES1), is induced by TGF- $\beta$ canonical signaling via SMADs $(9,10)$. Another study reported that Notch signaling also contributes 
to TGF- $\beta$-induced expression of a-SMA and COLI and, when inhibited, a-SMA and COLI expression decreased (11). Furthermore, the present research group has observed that overexpression of HES1 in the activated HSCs can enhance the promoter activity of $\alpha$-SMA and COLI 22 (12). Taken together, these findings indicate that there may exist a positive feedback loop between Notch and TGF- $\beta$ canonical signaling. Other experiments have already confirmed that HES1 can upregulate the expression of COLI $\alpha 1$ and COLI $\alpha 2$ on other cell types, such as fibroblast L929 cells and MRC-5 cells (13). Therefore, this positive feedback loop between Notch and TGF- $\beta$ canonical signaling may participate in HSC activation and hepatic fibrosis, with HES1 serving as an important TF in this crosstalk. HES1 belongs to the family of bHLH TFs, which contain the bHLH motif; this motif consists of $\sim 60$ amino acids, with a basic region and a helix 1-loop-helix 2, and the length of the loop differs between bHLH proteins (14). The bHLH protein family may be subdivided into three classes, according to their structure and biochemical characteristics, and HES1 belongs to the class $C$ proteins, which bind to the class $C$ DNA-binding domain (CACGNG) $(15,16)$. Furthermore, the authors of the present study discovered that abundant Class $\mathrm{C}$ binding domains in the promotor region of pro-fibrotic genes, include TGF- $\beta$, COLI $\alpha 1$, COLI $\alpha 2$, TIMP1, $\alpha$-SMA and Hes1 by the JASPAR database. The existence of Class $\mathrm{C}$ binding domains indicate DNA-protein interaction between the pro-fibrotic genes and Class $\mathrm{C}$ proteins may contribute to the activation of HSC and hepatic fibrosis.

Decoy oligodeoxynucleotides (ODNs) are also known as a TF 'trap'. Short-chain DNA fragments containing the DNA-binding site of specific TFs are artificially designed, synthesized and transfected to competitively capture intracellular TFs, thereby inhibiting downstream gene expression (17-19). The present study hypothesized that competitively inhibiting Class $\mathrm{C}$ proteins binding by Decoy ODN strategy may downregulate expression of TGF- $\beta$, COLI $\alpha 1$, COLI $\alpha 2$, TIMP1, $\alpha$-SMA and Hes1 and consequently inhibit HSC activation and relieve liver fibrosis.

\section{Materials and methods}

Synthesis of ODNs and plasmid construction. The decoy ODNs and scramble (Scr) decoy ODN (Table I) were synthesized by Sangon Biotech Co., Ltd. The eukaryotic expression plasmid pGLuc-TRE-MiniTK was constructed when TGF- $\beta$-responsive element (TRE) was cloned into the pGLuc-Mini-TK (New England Biolabs, Inc.). The eukaryotic expression plasmids pGLuc-P-SMA, pGLuc-P-COLI $\alpha 1$, pGLuc-P-COLI $\alpha 2$ and pGLuc-P-TIMP1 were constructed when the promoters of $\alpha$-SMA (P-SMA), COLI $\alpha 1$ (P-COLI $\alpha 1$ ), COLI 22 (P-COLI $\alpha 2)$ and TIMP1 (P-TIMP1) were cloned into the pGLucBasic vector (N8082S; New England Biolabs, Inc.) for luciferase assays (Table II).

Cell culture. HSC-T6 cells, an immortalized rat HSC line provided by the Huazhong University of Science and Technology, were cultured in high-glucose DMEM (Invitrogen; Thermo fisher Scientific, Inc.) supplemented with $10 \%$ newborn calf serum (Zhejiang Tianhang Biotechnology Co., Ltd). HSC-T6 cells were seeded at a density of
$6 \times 10^{5}$ cells/well in a 6-well-plate (Greiner $\left.\mathrm{GmbH}\right)$ for western blot assays or a 24-well-plate (Greiner $\mathrm{GmbH}$ ) for luciferase assays at $60 \%$ confluence per well and cultured in a humidified atmosphere containing $5 \% \mathrm{CO}_{2}$ for $24 \mathrm{~h}$ at $37^{\circ} \mathrm{C}$.

Transfection and luciferase reporter assays. The HSC-T6 cells were seeded at a density of $1 \times 10^{5}$ cells/well in a 24 -well plate. After $24 \mathrm{~h}$, the cells had reached $70-80 \%$ confluence and were transfected with different plasmids ( $1 \mu \mathrm{g}$ per well) using the Tubofect Transfection Reagent (Thermo Fisher Scientific, Inc.), according to the manufacturer's protocol. The cells were then transfected with different class $\mathrm{C}(\mathrm{C} 1 / \mathrm{C} 2 / \mathrm{C} 3 / \mathrm{C} 4)$ decoy ODNs and Scr decoy ODN (at a concentration of $20 \mathrm{~nm} / \mathrm{l}$; $2 \mu \mathrm{g}$ per well for a 24-well plate), using the Mirus Transfection Reagent (Mirus Bio LLC) after a further $24 \mathrm{~h}$. The luciferase assays were performed using the BioLux ${ }^{\circledR}$ Gaussia Luciferase Assay kit (New England Biolabs, Inc.), according to the manufacturer's protocol. Briefly, the supernatants were collected, the cells were lysed, and the total intracellular protein concentration of the supernatant was analyzed as described in the paragraph entitled 'Western blotting' of the Materials and methods section to estimate the cell number per well. For normalization, the sampling size for each well was adjusted according to the total intracellular protein levels to detect the Gaussia Luciferase activity. The reactions were examined using a fluorescence detector (Berthold Technologies).

Western blot analysis. The cells were collected for western blot assays after decoy ODNs (at a concentration of $20 \mathrm{~nm} / 1 ; 6 \mu \mathrm{g}$ per well for a 6-well plate) were transfected into HSC-T6 cells for $48 \mathrm{~h}$, then lysed in lysis buffer $[25 \mathrm{mmol} / \mathrm{l}$ Tris- $\mathrm{HCl}(\mathrm{pH} 7.5)$, $2.5 \mathrm{mmol} / 1 \mathrm{EDTA}, 137 \mathrm{mmol} / 1 \mathrm{NaCl}, 2.7 \mathrm{mmol} / 1 \mathrm{KCl}, 1 \%$ sodium deoxycholic acid, $0.1 \%$ SDS, $1 \%$ Triton X-100, and $2 \mathrm{mmol} / 1$ PMSF] and protease inhibitor cocktail for $30 \mathrm{~min}$ at $4^{\circ} \mathrm{C}$. Cell lysates were cleared by centrifugation at 7,200 $\mathrm{x}$ g for $10 \mathrm{~min}$ at $4^{\circ} \mathrm{C}$ and the supernatants were collected. Protein concentration was measured using a BCA Protein Assay kit (Thermo Fisher Scientific, Inc.). An equal amount of protein (40 $\mu \mathrm{g}$ loaded per lane) from each sample was separated by $10 \%$ SDS-PAGE and transferred to a PVDF membrane. The membrane was firstly incubated with blocker ( $5 \%$ defatted milk) for $2 \mathrm{~h}$ at room temperature and subsequently incubated with the following antibodies at $4^{\circ} \mathrm{C}$ overnight: Anti-TGF- $\beta 1$ (1:1,000; cat. no. sc-146; Santa Cruz Biotechnology, Inc.), anti-TIMP1(1:1,000; cat. no. sc-6834; Santa Cruz Biotechnology, Inc.), anti-COLI $\alpha 1$ (1:1,000; cat. no. sc-25974; Santa Cruz Biotechnology, Inc.), anti-COLI $\alpha 2$ (1:1,000; cat. no. sc-8788; Santa Cruz Biotechnology, Inc.), anti-SMAD3 (1:3,000; cat. no. sc-133098; Santa Cruz Biotechnology, Inc.) and anti- $\beta$-actin (1:3,000; cat. no. sc-47778; Santa Cruz Biotechnology, Inc.). Following the primary antibody incubation, membranes were incubated with horseradish peroxidase-conjugated secondary antibodies $(1: 3,000$; cat nos. sc-2031 and sc-516721; 1:8,000; cat. no. sc-2354; Santa Cruz Biotechnology, Inc.) for $1 \mathrm{~h}$ at room temperature. The membranes were treated using Immobilon Western Detection Reagents (EMD Millipore). Chemiluminescence was detected using the VersaDoc system (Bio-Rad Laboratories, Inc.). Densitometric analyses of the band intensities were performed using ImageJ software, version 1.38 (National Institutes of Health). All the western blot analysis were repeated three times. 
Table I. The sequences of each Decoy ODN.

\begin{tabular}{ll} 
Decoy ODN name & \multicolumn{1}{c}{ Sequence $\left(5^{\prime} \rightarrow 3^{\prime}\right)^{\mathrm{a}}$} \\
\hline Class C1 Decoy ODN & $\begin{array}{l}\text { F: CGACACGTGATCACGTGGAC } \\
\text { R: GTCEACGTGATCACGTGTCG }\end{array}$ \\
Class C2 Decoy ODN & $\begin{array}{l}\text { F: CGACACGCGATCACGCGGAC } \\
\text { R: GTCCGCGTGATCGCGTGTCG }\end{array}$ \\
Class C3 Decoy ODN & F: CGACACGAGATCACGAGGAC \\
& R: GTCCTCGTGATCTCGTGTCG \\
Class C4 Decoy ODN & F: CGACACGGGATCACGGGGAC \\
& R: GTCCCCGTGATCCCGTGTCG \\
Scramble & F: CGAACGCTGATACGCTGGAC \\
& R: GTCCAGCGTATCAGCGTTCG
\end{tabular}

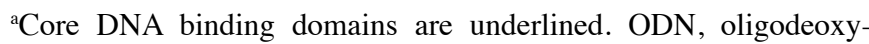
nucleotide; F, forward; $\mathrm{R}$, reverse.

Bioinformatics analysis. The JASPAR 2020 database (http://jaspar.genereg.net) and UCSC Genome Browser Gateway (http://genome.ucsc.edu/cgi-bin/hgGateway) were used for the bioinformatics analysis. Full-length Promoter sequences of $\alpha$-SMA, COLI $\alpha 1$, COLI $\alpha 2$ and TIMP1 were identified by UCSC Genome Browser Gateway. Detailed information of Class C TFBS (Basic helix-loop-helix factors) were identified by the JASPAR database. The distribution of Class C TFBS on Promoters of $\alpha$-SMA, COLI $\alpha 1$, COLI $\alpha 2$ and TIMP1 were analyzed by the JASPAR database.

Statistical analysis. GraphPad Prism version 7.0 software (GraphPad Software, Inc.) was used for the statistical analysis. Data are presented as the mean \pm SD and represent three independent experimental repeats. Differences between three or more groups were analyzed by one-way ANOVA and Tukey's post hoc test for multiple comparisons. $\mathrm{P}<0.05$ was considered to indicate a statistically significant difference.

\section{Results}

The class $C$ sequence is present in the promoter region of TGF- $\beta$ and its target genes. The JASPAR database is one of the most comprehensive and reliable public databases of TFs and DNA-binding motifs, and the data published there are rigorously screened from multiple randomized experiments and integrated by computer-aided software. This database was used in the present study to analyze the binding potency between the promoters of TGF- $\beta$ signaling pathway-related genes and class $\mathrm{C}$ sequences. The present study found at least one class $\mathrm{C}$ sequence that was present in the promoter region of TGF- $\beta$ and its downstream genes, namely COLI $\alpha 1, T I M P 1$, HESI and $\alpha$-SMA (Table III).

Class $C$ decoy ODNs decrease TGF- $\beta$ synthesis in HSC-T6 cells. The bioinformatics analysis revealed that there was at least one binding site in the promoter region of $T G F-\beta$ for each class $\mathrm{C}$ sequence. Class $\mathrm{C}$ decoy ODNs were transfected into
HSC-T6 cells for $48 \mathrm{~h}$ and the expression of $T G F-\beta$ was tested through western blot assays. Except for class C4 decoy ODNs, which had no impact on $T G F-\beta$ expression, the other three decoy ODNs were able to significantly downregulate $T G F-\beta$ expression $(\mathrm{P}<0.05$; Fig. 1A), indicating that class $\mathrm{C} 1 / \mathrm{C} 2 / \mathrm{C} 3$ decoy ODNs can decrease TGF- $\beta$ synthesis in HSC-T6 cells. Furthermore, plasmid pTRE-Mini-TK-Gluc, the Gaussia luciferase reporter gene for TRE was constructed and transfected into HSC-T6 cells for $24 \mathrm{~h}$; decoy ODNs were transfected for another $24 \mathrm{~h}$ and the results revealed that the luciferase activities of the pTRE-Mini-TK-Gluc had significantly decreased in all class $\mathrm{C}$ decoy ODN groups compared with $\mathrm{Scr}(\mathrm{P}<0.001$; Fig. 1B), suggesting the four class $\mathrm{C}$ decoy ODNs can inhibit the transcriptional activity of TRE.

Class $C$ decoy ODNs downregulate the expression of TIMPI in HSC-T6 cells. TIMP1 is a downstream gene of TGF- $\beta$ (4). A total of five binding sites for class $\mathrm{C}$ proteins in the promoter of TIMP1 were identified through bioinformatics analysis. The Gaussia luciferase activities of pTIMP1-GLuc-Basic significantly decreased in the four experimental groups treated by Class C1-4 decoy ODNs compared with $\mathrm{Scr}(\mathrm{P}<0.001$; Fig. 2A), suggesting the four decoy ODNs can inhibit the activation of the TIMP1 promoter. The expression of TIMP1 was also tested using western blot assays. There were significant decreases in TIMP1 expression in the four class $\mathrm{C}$ decoy ODN groups compared with Scr (Fig. 2B).

Class C decoy ODNs downregulate the expression of COLI $\alpha 1$ in HSC-T6 cells. COLI $\alpha 1$ is one of the downstream genes of TGF- $\beta$ and it is positively correlated with TGF- $\beta$ (4). Bioinformatics analysis demonstrated that there are five binding sites for class $\mathrm{C}$ sequences in its promoter. The results of the Gaussia luciferase assay revealed that, in the class $\mathrm{C} 1$ and class $\mathrm{C} 4$ decoy ODN groups, luciferase activity decreased compared with the Scr (Fig. 3A). The expression of COLI $\alpha 1$ was also tested using western blot assays, but only class $\mathrm{C} 1$ decoy ODNs were proven to downregulate the expression of COLI $\alpha 1$ (Fig. 3B).

Class $C$ decoy ODNs do not affect COLIa2 expression in HSC-T6 cells. COLI $\alpha 2$ is also regulated by the TGF- $\beta$ signaling pathway (4). Using a Gaussia luciferase assay, none of the four class $\mathrm{C}$ decoy ODNs were found to exert any effect on COLI $\alpha 2$ promoter activity (Fig. 4A). In addition, it was also confirmed that the four class $\mathrm{C}$ decoy ODNs exerted no effect on COLI $\alpha 2$ expression in HSC-T6 cells by western blotting (Fig. 4B).

Class C decoy ODNs downregulate $\alpha$-SMA and HES1 promoter activity. Previous studies have demonstrated that TGF- $\beta$ regulates the expression of $\alpha$-SMA and HES1 (20-23). Bioinformatics analysis revealed that there are five and ten binding sites for class $C$ sequences in the promoters of $\alpha$-SMA and HES1, respectively. The Gaussia luciferase activities of pSMA-GLuc-Basic were found to be significantly decreased in all experimental groups compared with $\mathrm{Scr}(\mathrm{P}<0.001$; Fig. 5A), suggesting that the four class $C$ decoy ODNs can downregulate the activity of the $\alpha$-SMA promoter, whereas only class $\mathrm{C} 1$ and class $\mathrm{C} 2$ decoy ODNs affect the activity of the HES1 promoter (Fig. 5B). 
Table II. Primers used for promoter cloning into pGLucBasic vector.

Gene Primer sequence $\left(5^{\prime} \rightarrow 3^{\prime}\right)$

P-Hairy and Enhancer of Split 1 (HindIII)

F: CGAAGCTTGAGCCTGAAGAGGTAGAGAGT

$\mathrm{P}-\alpha$-smooth muscle actin $(E c o \mathrm{R} 1)$

R: ATGGATCCGCTTACGTCCCCTTTACTTGG

F: CCGGAATTCACGGTCCTTAAGCATGATATC

R: CGGGATCCCTTACCCTGATGGCGACT

P-type I collagen $\alpha 1$ (EcoR1)

F: CCGGAATTCGCAGGTTCTCTACAGAGAGA

R: CGGGATCCAGCCAATCAGAACT

P-tissue inhibitor of metalloproteinase 1 (EcoR1)

F: GCGGAATTCCAAACATCTTCACTGGTATG
R: GCGGGATCCCTTTACTGGAAGCTATCAATG

$\mathrm{P}$, promotor; F, forward; R, reverse.

Table III. Analysis of the possible binding sites on the promoters of TGF- $\beta$ signal pathway-related genes for four class C sequences by JASPAR database.

\begin{tabular}{|c|c|c|c|c|c|}
\hline \multirow[b]{2}{*}{ Gene promoter } & \multicolumn{4}{|c|}{ Class } & \multirow[b]{2}{*}{ Total } \\
\hline & $\mathrm{C} 1$ & $\mathrm{C} 2$ & $\mathrm{C} 3$ & $\mathrm{C} 4$ & \\
\hline Transforming growth factor- $\beta$ & 1 & 6 & 1 & 3 & 11 \\
\hline Type I collagen $\alpha 1$ & 5 & 1 & 2 & 1 & 5 \\
\hline Type I collagen $\alpha 2$ & 0 & 0 & 0 & 0 & 0 \\
\hline Tissue inhibitor of metalloproteinase 1 & 0 & 0 & 2 & 3 & 5 \\
\hline Hairy and Enhancer of Split 1 & 4 & 1 & 3 & 2 & 5 \\
\hline$\alpha$-smooth muscle actin & 1 & 0 & 3 & 1 & 5 \\
\hline
\end{tabular}
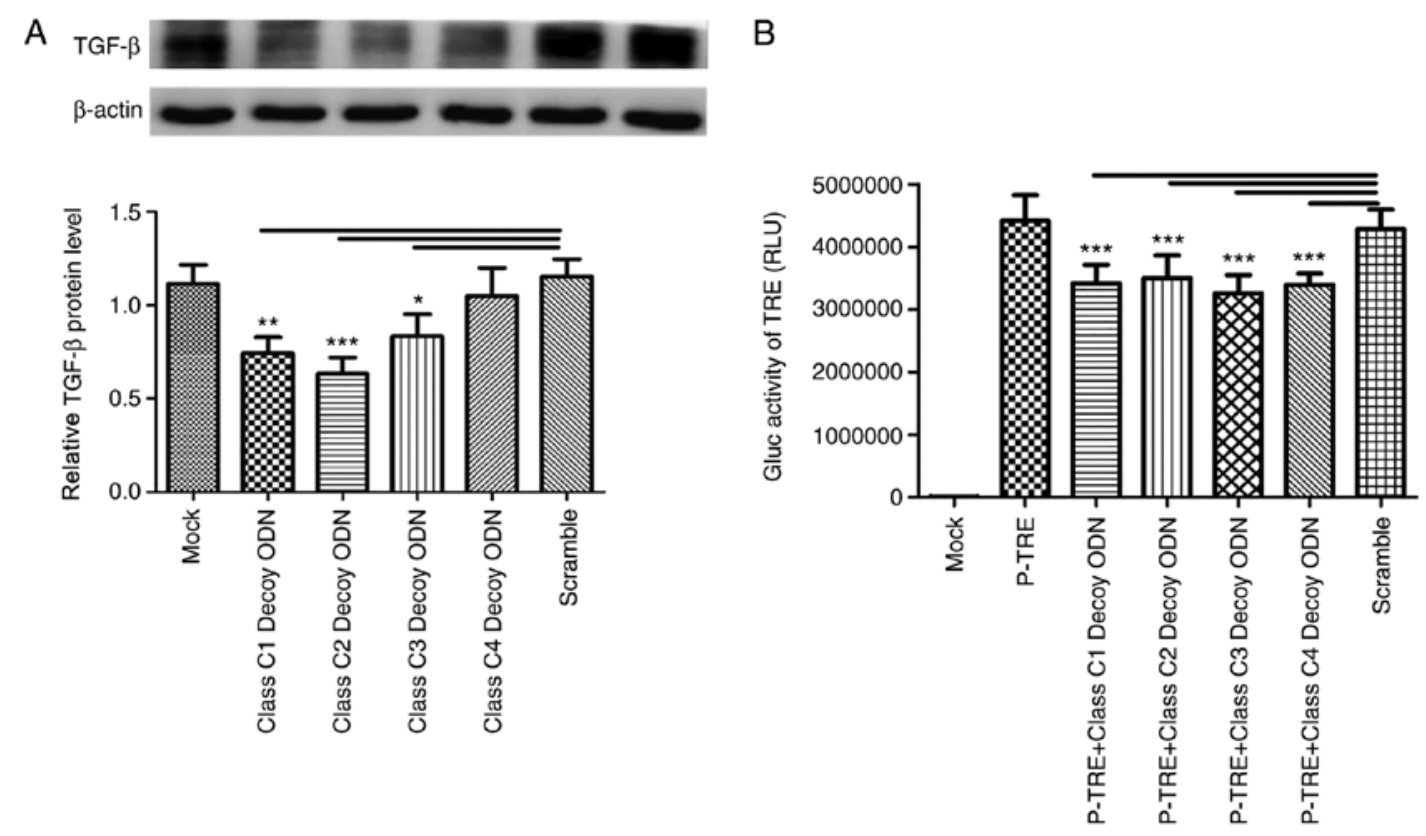

Figure 1. Influence of class C Decoy ODNs on TGF- $\beta$ and TRE in HSC-T6 cells. (A) Influence of four class C Decoy ODNs on the expression of TGF- $\beta$ by western blot assays in HSC-T6 cells. The $\beta$-actin protein served as a control. Scramble served as control. Quantification of TGF- $\beta$ expression in HSC-T6 cells by western blot showed significant decreases in class C1 Decoy ODN, class C2 Decoy ODN and class C3 Decoy ODN group compared with scramble control, respectively. ${ }^{*} \mathrm{P}<0.05,{ }^{* *} \mathrm{P}<0.01$ and ${ }^{* * *} \mathrm{P}<0.001$. Data are presented as the mean $\pm \mathrm{SD}$ of three individual experiments. The band intensities were normalized to $\beta$-actin in the quantitative analysis. (B) Influence of four class C Decoy ODNs on the activity of TRE in HSC-T6 cells. After pGLuc-TRE-MiniTK was transfected into HSC-T6 cells for $24 \mathrm{~h}$, Decoy ODNs were transfected for another $24 \mathrm{~h} .{ }^{* * *} \mathrm{P}<0.001$. Data are presented as the mean \pm standard deviation of three individual experiments. SD, standard deviation; TGF, transforming growth factor; ODN, oligodeoxynucleotides; P-TRE, TGF- $\beta$-responsive element promoter; RLU, relative light units. 
A

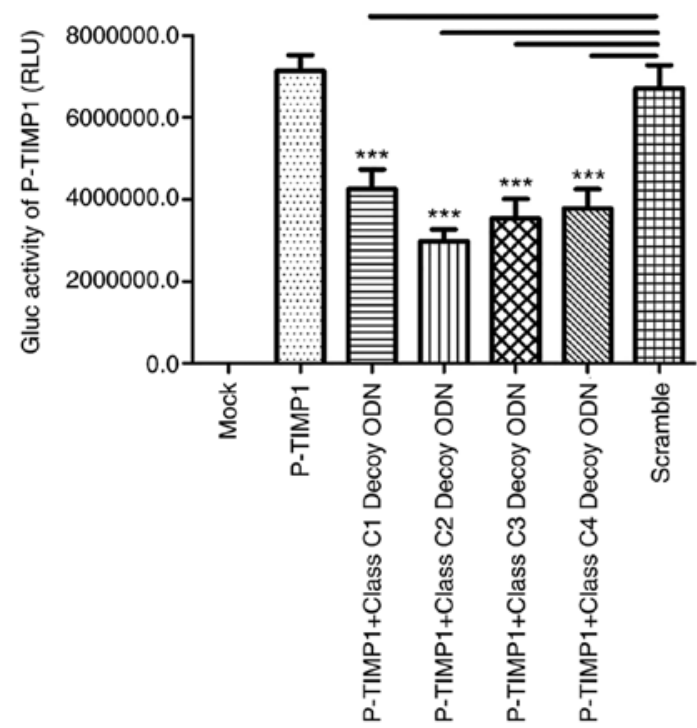

$\mathrm{B}_{\text {TIMP1 }}$

$\beta$-actin


Figure 2. Influence of four class C Decoy ODNs on TIMP1 in HSC-T6 cells. (A) Luciferase reporter assays of four class C Decoy ODNs on the activity of TIMP1 promoter in HSC-T6 cells. After pTIMP1-GLuc-Basic was transfected into HSC-T6 cells for $24 \mathrm{~h}$, four class C Decoy ODNs were transfected for another $24 \mathrm{~h}$. Scramble group served as control. ${ }^{* * *} \mathrm{P}<0.001$. Data are presented as the mean $\pm \mathrm{SD}$ of three individual experiments. (B) Influence of four class $\mathrm{C}$ Decoy ODNs on the expression of TGF- $\beta$ by western blot assays in HSC-T6 cells. The $\beta$-actin protein served as control. Scramble served as control. Quantification of TGF- $\beta$ expression in HSC-T6 cells by western blotting showed significant decreases in four class C Decoy ODNs groups compared with scramble control, respectively. ${ }^{* * * *} \mathrm{P}<0.001$. Data are presented as the mean $\pm \mathrm{SD}$ of three experiments. The band intensities were normalized to $\beta$-actin in the quantitative analysis SD, standard deviation; P-TIMP, promoter tissue inhibitor of matrix metalloproteinase; ODNs, oligodeoxynucleotides; TGF, transforming growth factor; RLU, relative light units.

A

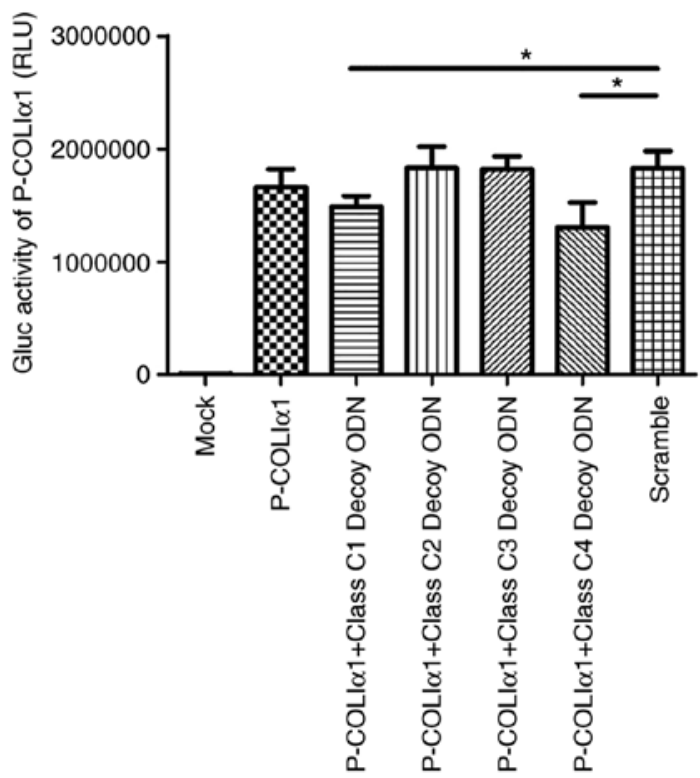

B
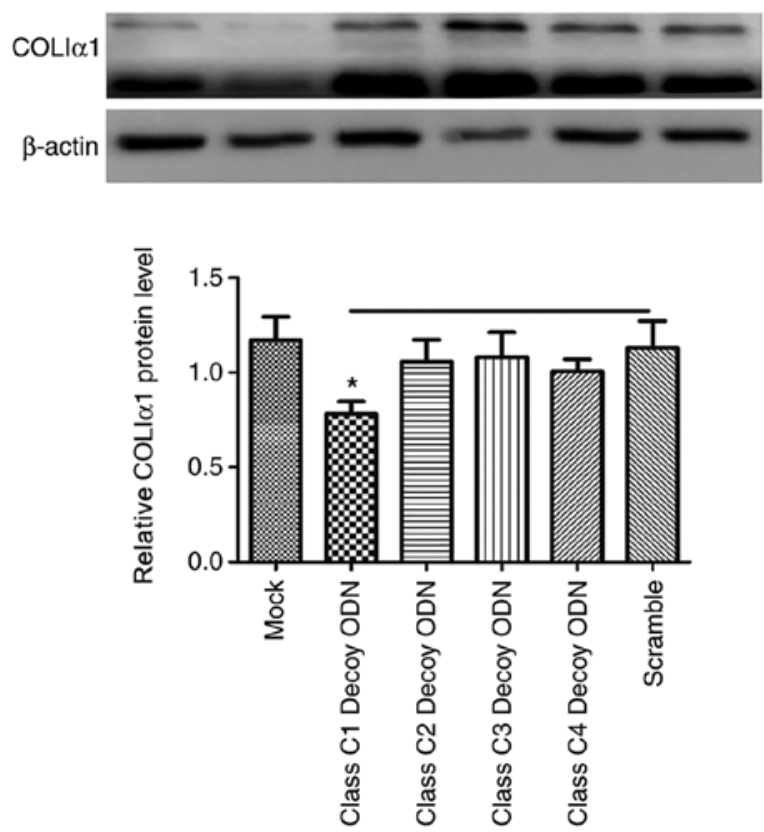

Figure 3. Influence of four class C Decoy ODNs on COLI 1 in HSC-T6 cells. (A) Luciferase reporter assays of four class C Decoy ODNs on the activity of COLI $\alpha 1$ promoter in HSC-T6 cells. After pCOLI $\alpha 1$-GLuc-Basic was transfected into HSC-T6 cells for $24 \mathrm{~h}$, four class C Decoy ODNs were transfected for another $24 \mathrm{~h}$. The Scramble group served as a control. "P<0.05. Data are presented as the mean \pm SD of eight wells. (B) Influence of four class C Decoy ODNs on the expression of COLI $\alpha 1$ by western blot assays in HSC-T6 cells. The two protein bands represent COL1A1 and COL1A1 precursor respectively, in accordance with the protocol of the COL1A1 antibody. The $\beta$-actin protein served as a control. The Scramble group also served as a control. Quantification of COLI $\alpha 1$ expression in HSC-T6 cells by western blotting showed a significant decrease only in the class C1 Decoy ODN group compared with the Scramble control group. ${ }^{*} \mathrm{P}<0.05$. Data are presented as the mean $\pm \mathrm{SD}$ of three experiments. The band intensities were normalized to $\beta$-actin in the quantitative analysis. $\mathrm{SD}$, standard deviation; COL, collagen; ODN, oligodeoxynucleotides; RLU, relative light units. 

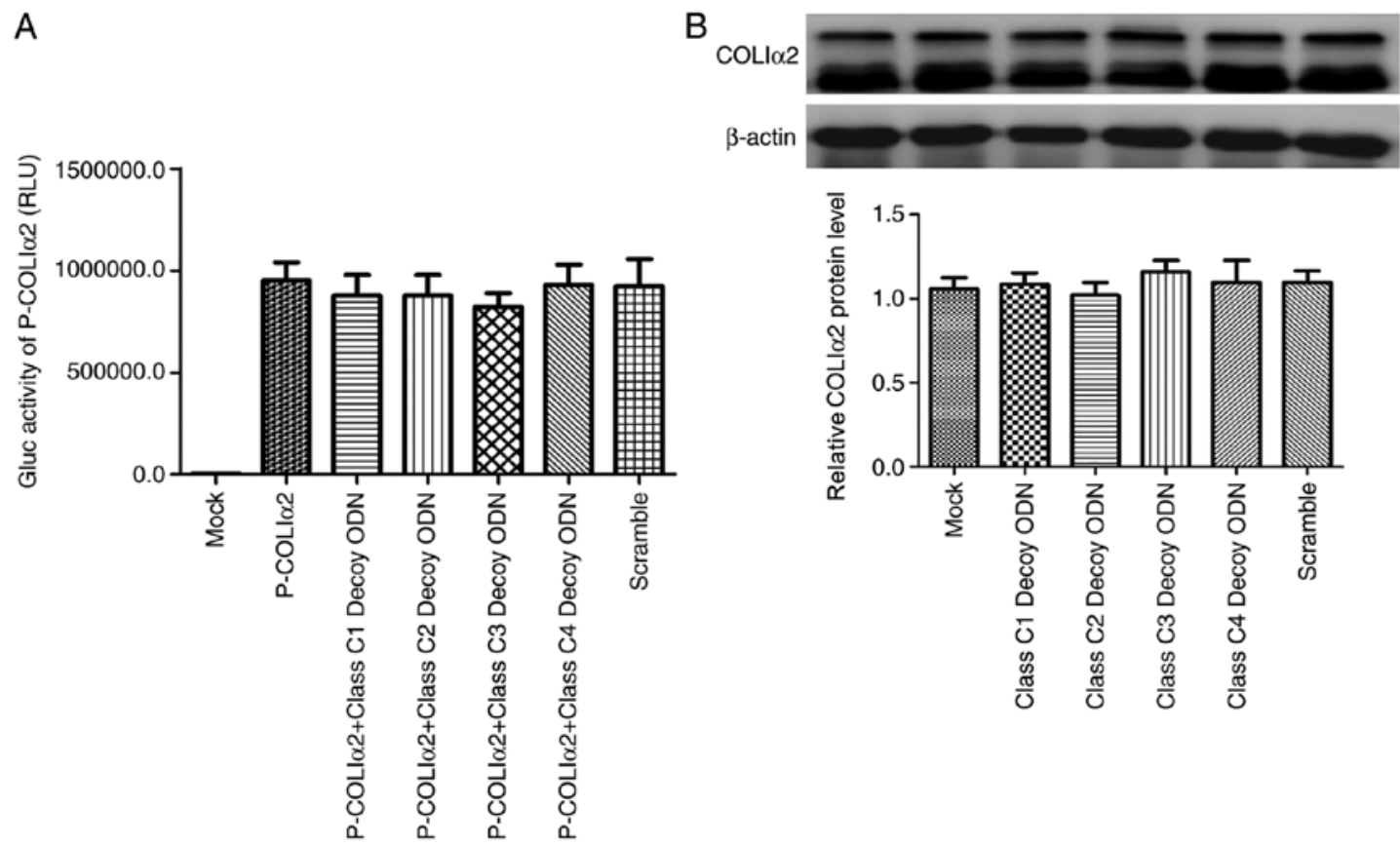

Figure 4. Influence of four class C Decoy ODNs on COLI $\alpha 2$ in HSC-T6 cells. (A) Luciferase reporter assays of four class C Decoy ODNs on the activity of COLIa2 promoter in HSC-T6 cells. After pCOLI $\alpha 2-$ GLuc-Basic was transfected into HSC-T6 cells for $24 \mathrm{~h}$, four class C Decoy ODNs were transfected for another $24 \mathrm{~h}$. The Scramble group served as a control. Data are presented as the mean \pm SD of three individual experiments. (B) The influence of four class C Decoy ODNs on the expression of COLI $\alpha 2$ by western blot assays in HSC-T6 cells. Two protein bands represent the COL1A2 and COL1A2 precursor respectively, in accordance with the protocol of the COL1A2 antibody. The $\beta$-actin protein served as a control. The Scramble served as a control. Quantification of COLI $\alpha 2$ expression in HSC-T6 cells by western blot showed that four class C Decoy ODNs had no effect on COLI 22 expression in HSC-T6 cells. Data are presented as the mean \pm SD of three individual experiments. The band intensities were normalized to $\beta$-actin in the quantitative analysis. SD, standard deviation; COL, collagen; ODN, oligodeoxynucleotides; RLU, relative light units.
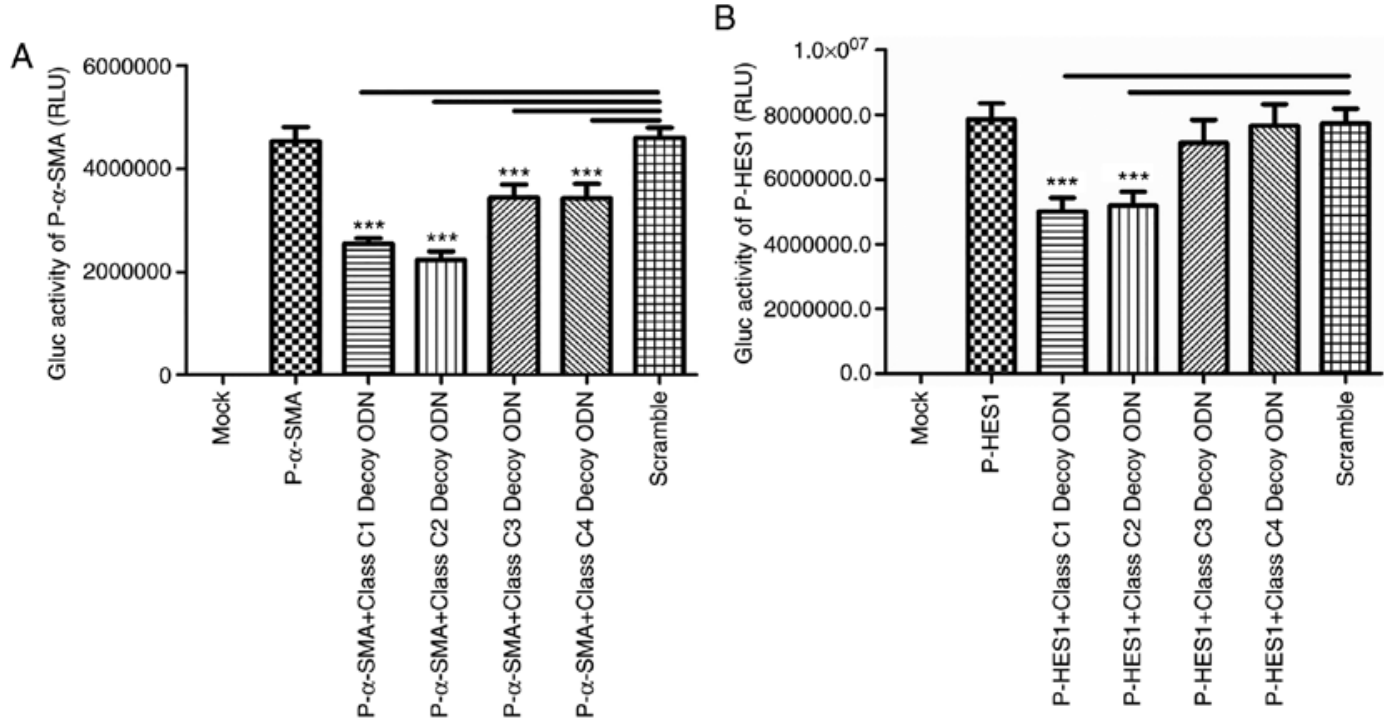

Figure 5. Influence of four class C Decoy ODNs on $\alpha$-SMA and HES1 in HSC-T6 cells. (A) Luciferase reporter assays of four class C Decoy ODNs on the activity of $\alpha$-SMA promoter in HSC-T6 cells. After pSMA-GLuc-Basic was transfected into HSC-T6 cells for $24 \mathrm{~h}$, four class C Decoy ODNs were transfected for another $24 \mathrm{~h}$. The Scramble group served as a control. Data are presented as the mean \pm SD of three individual experiments. (B) Luciferase reporter assays of four class C Decoy ODNs on the activity of HES1 promoter in HSC-T6 cells. After pHES1-GLuc-Basic was transfected into HSC-T6 cells for 24 h, four class C Decoy ODNs were transfected for another $24 \mathrm{~h}$. The Scramble group served as a control. ${ }^{* * *} \mathrm{P}<0.001$. Data are presented as the mean $\pm \mathrm{SD}$ of three individual experiments. SD, standard deviation; ODN, oligodeoxynucleotides; RLU, relative light units; P-SMA, promoter smooth muscle actin.

In conclusion, class $\mathrm{C} 1$ decoy ODNs exerted the most prominent effect on TGF- $\beta$ signaling pathway-related genes and it downregulated the expression of TGF- $\beta$, TIMP1, HES1, $\alpha$-SMA and COL1 $\alpha 1$.
Class C1 decoy ODNs downregulate SMAD3 expression. Class $\mathrm{C} 1$ decoy ODNs were found to exert the broadest and most prominent effect on TGF- $\beta$ signaling pathway-related genes, and it inhibited the promoter activity of TGF- $\beta$ and 


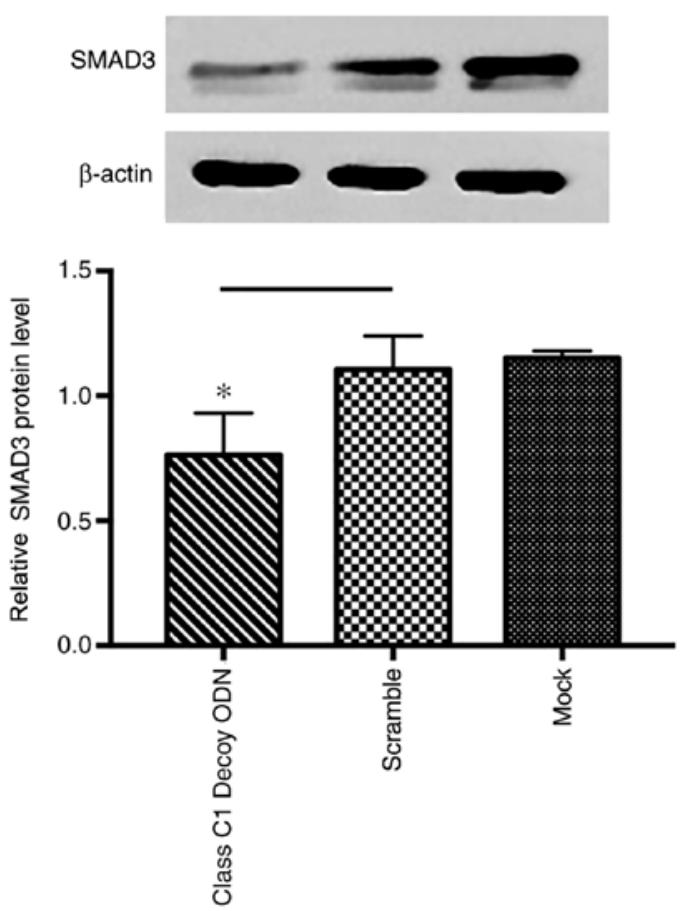

Figure 6. Influence of class C1 Decoy ODN on SMAD3 in HSC-T6 cells. Influence of class $\mathrm{C} 1$ Decoy ODN on the expression of SMAD3 by western blot assays in HSC-T6 cells. The $\beta$-actin protein served as a control. The Scramble served as a control. Quantification of TGF- $\beta$ expression in HSC-T6 cells by western blotting showed significant decreases in class C1 Decoy ODN group compared with the scramble control. " $\mathrm{P}<0.05$. Data are presented as the mean \pm standard deviation of three experiments. The band intensities were normalized to $\beta$-actin in the quantitative analysis. ODN, oligodeoxynucleotides.

its downstream target genes, namely COLI $\alpha 1$, TIMP1 and $\alpha$-SMA, and further downregulated the protein expression of TGF- $\beta$, COLI $\alpha 1$ and TIMP1. To investigate the mechanism through which class $\mathrm{C} 1$ decoy ODNs downregulated TGF- $\beta$ signaling pathway-related genes, the expression of COLI $\alpha 1$ and SMAD3 was tested using western blot assays and proven to be significantly downregulated by class $\mathrm{C} 1$ decoy ODNs $(\mathrm{P}<0.05$; Fig. 6).

\section{Discussion}

Liver fibrosis is an intermediate stage between primary liver disease, liver cirrhosis, or even liver cancer. Thus, reversing the process of liver fibrosis is key to preventing this life-threatening progression. The major pathological characteristic associated with liver fibrosis is disruption of the balance between ECM synthesis and degradation $(1,2)$. Various cell-stimulating factors act on HSCs to promote their activation and proliferation. Through proliferation, secretion of ECM and contraction, activated HSCs are actively involved in the occurrence of liver fibrosis and intrahepatic structural remodeling, which is considered as the pathological basis of liver fibrosis and portal hypertension. TGF- $\beta 1$ is currently recognized as the strongest pro-fibrosis factor by stimulating HSCs (1-3), which mediate TGF- $\beta 1$ signals from the cytoplasm to the nucleus, ultimately inducing collagen (type I, II, III and others) synthesis and secretion. Furthermore, it may also promote secretion of TIMPs that can inhibit matrix metalloproteinases synthesis, resulting in ineffective collagen degradation. It is broadly accepted that TGF- $\beta 1$ canonical signaling, also known as the TGF- $\beta 1 /$ SMADs signaling pathway, is crucial for the occurrence and progression of hepatic fibrosis, whereas non-canonical signaling, which is associated with multiple different pathways, such as MAPK, PI3K-AKT and Wnt, also contributes to the activation of HSCs and liver fibrosis $(7,8)$. Several previous studies have revealed the existence of crosstalk between Notch and TGF- $\beta$ signaling in the activation of HSCs, and the Notch downstream TF HES1 plays an important role in this crosstalk (9-13). Thus, blocking the signal transduction of TGF- $\beta 1$ or regulating the effect of SMADs on the expression of target genes in order to decrease ECM synthesis and increase ECM degradation may be a promising approach to reversing hepatic fibrosis.

HES1 belongs to the highly conserved bHLH family of TFs, which are $\sim 60$ amino acids in length and named according to their $\beta$ helix-loop-helix structure. The C-type TF of the bHLH family serves a role as a homologous or heterodimeric form that binds to the class $\mathrm{C}$ sequence (CACGNG) (15). Bioinformatics analysis demonstrated that the CACGNG sequence was present on the promoter region of the TGF- $\beta 1$, COLI $\alpha 1$, TIMP1, HES1 and $\alpha$-SMA genes, indicating that the C-type TF of the bHLH family may modulate the expression of those pro-fibrotic genes. The results of the bioinformatics analysis were consistent with the literature review (11-13). Using the decoy ODN strategy, it was confirmed that class $\mathrm{C}$ decoy ODNs have different capacities of inhibiting the expression of pro-fibrotic genes, such as TGF- $\beta$, SMAD3, COLI $\alpha 1$ and TIMP1, and downregulating the transcriptional activity of the HES1 and $\alpha$-SMA promoters, as well as TRE. Among the four decoy ODNs, class $\mathrm{C} 1$ decoy ODNs, which carry a class C TF trap sequence (CACGTG), are the most efficient for downregulating those target genes, following by Class $\mathrm{C} 2$, which indicate Class C1\&2 DNA binding domains has a greater affinity for Class $C$ proteins than $C 3 \& 4$. It seemed to be paradoxical that by bioinformatic analysis, the binding site of Class $\mathrm{C} 3 \& 4$ are outnumbered compared with the binding site of Class C1\&2. This is especially true in the promotor region of Hes1, where Class1\&2 has 1 binding site and Class C3\&4 has 4 binding sites and in the promotor region of TIMP1, where Class1\&2 has 0 binding sites and Class C $3 \& 4$ has 5 binding sites. The present study assumed that the Class $\mathrm{C}$ proteins bind to Class $\mathrm{C} 3 \& 4$ binding sites in Hes 1 and the TIMP1 promoter, and after Class C1\&2 decoy ODN which carried a binding domain with better affinity was conducted into the cell, it captured Class $\mathrm{C}$ proteins and competitively inhibited their binding with the Class C3\&4 binding site in the reporter plasmid, and then the G-luciferase activity decreased. Similarly, Class C1\&2 can downregulate TIMP-1 promoter activity and its expression, which can also be explained by the strong affinity to TFs of exogenous Class C1\&2 sequence.

By reducing COLI $\alpha 1$ synthesis and promoting ECM degradation via downregulating TIMP1, as well as repressing HSC transactivation via downregulating TGF- $\beta$ and $\alpha$-SMA, class $\mathrm{C} 1$ decoy ODNs appear to be promising for preventing HSC activation and hepatic fibrosis. The possible mechanism underlying the anti-fibrotic effects of class $\mathrm{C} 1$ decoy ODNs is competitive binding of class C TFs, including HES1, or indirect repression by inhibiting the TGF- $\beta$ /SMADs pathway, as 
the synthesis of TGF- $\beta$ and SMAD3 was downregulated and the transcriptional activity of TRE was inhibited. However, the applicability of class $\mathrm{C} 1$ decoy ODNs in the clinical setting requires further investigation.

\section{Acknowledgements}

Not applicable.

\section{Funding}

The present study was supported by The National Natural Science Foundation of China (grant. no. 81670555), The Health Commission of Hubei Province Scientific Research Project (grant. no. WJ2019H533) and The Hubei Provincial Department of Education (grant. no. Q20181208).

\section{Availability of data and materials}

The datasets used and/or analyzed during the current study are available from the corresponding author on reasonable request.

\section{Authors' contributions}

JFW, LH and CBL conceived and designed the experiments; CR, YRN, YMZ, YQZ and RTZ performed the experiments and analyzed the data; CR and YRN wrote the manuscript. CBL was responsible for the language editing of the manuscript. All authors read and approved the final submitted version of the manuscript.

\section{Ethics approval and consent to participate}

Not applicable.

\section{Patient consent for publication}

Not applicable.

\section{Competing interests}

The authors declare that they have no competing interests.

\section{References}

1. Hernandez-Gea V and Friedman SL: Pathogenesis of liver fibrosis. Annu Rev Pathol 6: 425-456, 2011.

2. Novo E, Cannito S, Paternostro C, Bocca C, Miglietta A and Parola M: Cellular and molecular mechanisms in liver fibrogenesis. Arch Biochem Biophys 548: 20-37, 2014.

3. Yoshida K, Murata M, Yamaguchi T and Matsuzaki K: TGF- $\beta /$ Smad signaling during hepatic fibro-carcinogenesis (review). Int J Oncol 45: 1363-1371, 2014.

4. Sa Y, Li C, Li H and Guo H: TIMP-1 Induces $\alpha$-Smooth muscle actin in fibroblasts to promote urethral scar formation. Cell Physiol Biochem 35: 2233-2243, 2015.
5. Bi WR, Yang CQ and Shi Q: Transforming growth factor- $\beta 1$ induced epithelial-mesenchymal transition in hepatic fibrosis. Hepatogastroenterology 59: 1960-1963, 2012.

6. Okazaki I, Noro T, Tsutsui N, Yamanouchi E, Kuroda H, Nakano M, Yokomori H and Inagaki Y: Fibrogenesis and carcinogenesis in non-alcoholic steatohepatitis (NASH): Involvement of matrix metalloproteinases (MMPs) and tissue inhibitors of metalloproteinase (TIMPs). Cancers Basel) 6: 1220-1255, 2014.

7. Poelstra K: Liver fibrosis in 2015: Crucial steps towards an effective treatment. Nat Rev Gastroenterol Hepatol 13: 67-68, 2016.

8. Tacke F and Trautwein C: Mechanisms of liver fibrosis resolution. J Hepatol 63: 1038-1039, 2015

9. Wang Y, Shen RW, Han B, Li Z, Xiong L, Zhang FY, Cong BB and Zhang B: Notch signaling mediated by TGF- $\beta / \mathrm{Smad}$ pathway in concanavalin A-induced liver fibrosis in rats. World J Gastroenterol 23: 2330-2336, 2017.

10. Aimaiti Y, Jin X, Wang W, Chen Z and Li D: TGF- $\beta 1$ signaling regulates mouse hepatic stellate cell differentiation via the Jagged1/Notch pathway. Life Sci 192: 221-230, 2018.

11. Chen YX, Weng ZH, Qi D and Zhang SL: Effect of Notch signaling on the activation of hepatic stellate cells. Zhonghua Gan Zang Bing Za Zhi 20: 677-682, 2012 (In Chinese).

12. Zhang K, Zhang YQ, Ai WB, Hu QT, Zhang QJ, Wan LY, Wang XL, Liu CB and Wu JF: Hes1, an important gene for activation of hepatic stellate cells, is regulated by Notch1 and TGF- $\beta$ /BMP signaling. World J Gastroenterol 21: 878-887, 2015.

13. Hu M, Ou-Yang HF, Wu CG, Qu SY, Xu XT and Wang P: Notch signaling regulates coll $\alpha 1$ and coll $\alpha 2$ expression in airway fibroblasts. Exp Biol Med (Maywood 239: 1589-1596, 2014.

14. Ma PC, Rould MA, Weintraub H and Pabo CO: Crystal structure of MyoD bHLH domain-DNA complex: Perspectives on DNA recognition and implications for transcriptional activation. Cell 77: 451-459, 1994.

15. Iso $T$, Kedes $L$ and Hamamori Y: HES and HERP families: Multiple effectors of the Notch signaling pathway. J Cell Physiol 194: 237-255, 2003.

16. Kobayashi T and Kageyama R: Expression dynamics and functions of Hes factors in development and diseases. Curr Top Dev Biol 110: 263-283, 2014.

17. Tomita N, Ogihara T and Morishita R: Transcription factors as molecular targets: Molecular mechanisms of decoy ODN and their design. Curr Drug Targets 4: 603-608, 2003.

18. Tomita N, Azuma H, Kaneda Y, Ogihara T and Morishita R: Gene therapy with transcription factor decoy oligonucleotides as a potential treatment for cardiovascular diseases. Curr Drug Targets 4: 339-346, 2003.

19. Jia D, Ni YR, Zhang YQ, Rao C, Hou J, Tang HQ, Liu CB and Wu JF: SP1 and UTE1 Decoy ODNs inhibit activation and proliferation of hepatic stellate cells by targeting tissue inhibitors of metalloproteinase 1. Cell Biosci 6: 31, 2016.

20. Krizhanovsky V, Yon M, Dickins RA, Hearn S, Simon J, Miething C, Yee H, Zender L and Lowe SW: Senescence of activated stellate cells limits liver fibrosis. Cell 134: 657-667, 2008.

21. Li YH, Woo SH, Choi DH and Cho EH: Succinate causes $\alpha-S M A$ production through GPR91 activation in hepatic stellate cells. Biochem Biophys Res Commun 463: 853-858, 2015.

22. Blokzijl A, Dahlqvist C, Reissmann E, Falk A, Moliner A, Lendahl U and Ibáñez CF: Cross-talk between the Notch and TGF-beta signaling pathways mediated by interaction of the Notch intracellular domain with Smad3. J Cell Biol 163: 723-728, 2003.

23. Liu L, Gao C, Chen G, Li X, Li J, Wan Q and Xu Y: Notch signaling molecules activate TGF- $\beta$ in rat Mesangial cells under high glucose conditions. J Diabetes Res 2013: 979702, 2013.

This work is licensed under a Creative Commons Attribution-NonCommercial-NoDerivatives 4.0 International (CC BY-NC-ND 4.0) License. 